\title{
Porcher, Jocelyne/Estebanez, Jean (Hrsg.): Animal Labor. A New Perspective on Human-Animal Relations, 182 S., transcript, Bielefeld 2019.
}

\section{Mieke Roscher}

Online publiziert: 23. Juli 2020

(C) Der/die Autor(en) 2020

2007 begründete der amerikanische Sozialwissenschaftler Jason Hribal in einem Artikel für die Zeitschrift Human Ecology Review seine Forderung nach der Anerkennung tierlicher Agency mit der Arbeit, die Tiere leisten würden. Auch Donna Haraway sprach in ihrem Buch „When Species Meet“ (2013) im Zusammenhang mit Versuchstieren von einer ,shared condition of work“, um Handeln jenseits des Menschen zu fassen. Agency, die Handlungs- und Wirkmacht von Tieren, hat seitdem eine enorme Strahlkraft innerhalb der sozialwissenschaftlichen Tierforschung entfaltet. Das Konzept von Arbeit interessanterweise nicht. Ein Versuch, diese Lücke zu füllen, unternimmt der Sammelband „Animal Labor“, herausgegeben von der Arbeitssoziologin Jocelyne Porcher und dem Kulturgeografen Jean Estebanez. Er präsentiert Ergebnisse eines von 2012 bis 2016 laufenden Forschungsprojektes zu Arbeitsbeziehungen mit Tieren im Produktions- und Servicesektor, das an verschiedenen französischen Universitäten durchgeführt wurde. Der Band umfasst zehn aus dem Französischen ins Englische übersetzte Aufsätze, die teilweise von den Herausgeber_innen mitverfasst wurden.

Mit ihrem einleitenden, programmatischen Aufsatz „Animal Labor“ verweigert sich Porcher gleich zu Beginn einer Erzählung, die Arbeit von Tieren als reine Ausbeutung und Unterdrückung definiert und hebt hier darauf ab, einerseits Arbeit als relationale Tätigkeit zu beschreiben und anderseits klare Grenzen zu ziehen. Massentierhaltung habe mit Arbeit nichts zu tun, weil nicht die Arbeit, sondern das Produkt im Mittelpunkt stünde. Mit ihrem relationalen Ansatz folgt sie aber weniger der neuen Sozialtheorie, als vielmehr einer ethisch-philosophischen Idee von gegenseitiger Achtung. Dies ist mitunter verwirrend. Einerseits spricht sie sich für eine ethische Berücksichtigung aus, um gleichzeitig dem Feld der Human-Animal Studies abolitionistische Züge zu- und damit quasi deren Wissenschaftlichkeit abzusprechen. Sie fordert ferner, einen Arbeitsbegriff anzuwenden, der sich jenseits des modernen kapitalistischen Systems anwenden ließe. Ihrem Team und ihr geht es insgesamt darum, zu zeigen, inwieweit Mensch und Tier durch Arbeit in ,asymmetrical yet reciprocal relationships“ (S. 27) aneinandergebunden sind und dass Tiere in ihrem Arbeiten nicht naturalisiert werden sollten: Tierliche Arbeit, so meinen

M. Roscher $(\square)$

Universität Kassel, Kassel, Deutschland

E-Mail: roscher@uni-kassel.de 
alle Autor_innen, finde im Sozialen statt. Die Beweise dafür sollen ethnografisch basierte Fallstudien liefern. Die Tiefenbohrungen beginnen jedoch nicht mit der Arbeit von Tieren, sondern von Robotern sowohl in der Produktionsindustrie als auch in jenem Feld, in dem Tiere Porcher zufolge schon immer Arbeit geleistet hätten: im emotionalen Arbeitssektor und in der Pflege. Dieser breite Einsatz von Robotern verdränge Tiere aus allen Bereichen herkömmlicher Tierarbeit, wie auch neue künstliche Nahrung das Tier als landwirtschaftliches Subjekt seiner Aufgabe der Fleischlieferung entbinde. Dieser Befund hätte Anlass für eine aufschlussreiche Diskussion sein können. In schon fast unerträglicher agrarromantisierender Fasson sehnt Porcher jedoch eine Welt vor der Industrialisierung des Agrarmarktes zurück, in der sich Mensch und Tier im gemeinsamen Schaffen als Subjekte konstituieren.

Sehr viel überzeugender ist Estelle Déleages Beitrag zur Technisierung des Agrarmarktes, in der sie Arbeit in kleineren und größeren Betrieben gegeneinanderstellt und hier mögliche Konsequenzen für die Arbeit als Prozess herausfiltert (Kap. 9). Es folgen Kapitel zu Pferden in Verhaltensexperimenten und Tieren in der Filmindustrie. Die spannende Frage, ob Belohnungssysteme eigentliche intrinsische Motivation befördern oder einem Lohnsystem gleichzustellen sind, wird hier mit dem tierlichen Anerkennungsbedürfnis beantwortet. Zudem sei Konditionierung allein nicht tragend für ,richtige " Arbeit, wobei Arbeit immer, und in jedem Aufsatz aufs Neue, als ,working is living together“ und als ein Set einzuhaltender Regeln definiert wird. Gänzlich fehlt in diesen ersten Aufsätzen eine genauere Beschreibung der Methode der Ethnografie. Das mag für Nicht-Sozialwissenschaftler_innen erleichternd wirken, eine solche wäre aber im Hinblick auf eine Weiterentwicklung der Multispezies-Ethnografie hilfreich gewesen. Dies wird zu einem gewissen Maße in den Fallstudien nachgeholt, so etwa im Beitrag von Nicolas Lainé zu den Arbeitsbeziehungen zwischen Elefanten und Menschen in Asien. Allerdings spielt die ethnografische Beobachtung der Tiere bei der Arbeit mit Menschen in den weiteren Kapiteln kaum eine Rolle, eher wird auf Interviews zurückgegriffen. Lainés überzeugender Beitrag zeichnet ein differenzierteres Bild der Arbeit, in dem nicht alle Tätigkeiten der Elefanten gleichsam als Arbeit definiert werden. Die Sozialisation eines Elefanten wird hier als komplexer - zwar nicht als gewaltfreier, aber auch nicht nur dominierender - Prozess gelesen, aus dem erst die Möglichkeit der Kollaboration erwächst. Die nächsten drei Aufsätze befassen sich mit Hunden in ihren Arbeitsbeziehungen als Blindenführhunde, Schutzhunde gegen Wölfe und im militärischen Dienst. Warum ihnen viel Aufmerksamkeit geschenkt wird, liegt auf der Hand. Ihre Sozialkompetenz zeigt sich auch im Arbeitsprozess. „Working“ als „living together“ überzeugt hier unmittelbar genauso wie die Argumente dafür, sowohl die in der Regel unfreie Arbeit von Tieren als auch für sie befriedigende Tätigkeiten zu klassifizieren und zwischen sichtbarer und eher unsichtbarer Arbeit zu unterscheiden.

Insgesamt bietet der Band einige sehr wichtige Denkanstöße für die weitere Debatte. Dennoch beschleicht die Rezensentin ein ausgesprochen ungutes Gefühl, wenn Porcher tierliche Arbeit mit Freiheit verbindet, was die Annahme zulässt, das Tier hätte sich erst durch die Domestikation befreit.

Funding Open Access funding provided by Projekt DEAL. 
Open Access Dieser Artikel wird unter der Creative Commons Namensnennung 4.0 International Lizenz veröffentlicht, welche die Nutzung, Vervielfältigung, Bearbeitung, Verbreitung und Wiedergabe in jeglichem Medium und Format erlaubt, sofern Sie den/die ursprünglichen Autor(en) und die Quelle ordnungsgemäß nennen, einen Link zur Creative Commons Lizenz beifügen und angeben, ob Änderungen vorgenommen wurden.

Die in diesem Artikel enthaltenen Bilder und sonstiges Drittmaterial unterliegen ebenfalls der genannten Creative Commons Lizenz, sofern sich aus der Abbildungslegende nichts anderes ergibt. Sofern das betreffende Material nicht unter der genannten Creative Commons Lizenz steht und die betreffende Handlung nicht nach gesetzlichen Vorschriften erlaubt ist, ist für die oben aufgeführten Weiterverwendungen des Materials die Einwilligung des jeweiligen Rechteinhabers einzuholen.

Weitere Details zur Lizenz entnehmen Sie bitte der Lizenzinformation auf http://creativecommons.org/ licenses/by/4.0/deed.de.

\section{Plöckinger, Othmar (Hrsg.): Sprache zwischen Politik, Ideologie und Geschichtsschreibung. Analysen historischer und aktueller Übersetzungen von ,Mein Kampf"“ (Beiträge zur Kommunikationsgeschichte, Bd. 32), 244 S., Steiner, Stuttgart 2019.}

\section{Wolfgang Schieder}

Online publiziert: 31. Juli 2020

(C) Gesellschaft zur wissenschaftlichen Förderung politischer Literatur e.V. and the Author(s) 2020

Es ist keine schlechte Idee, einmal Übersetzungen von Adolf Hitlers „Mein Kampf“ in andere Sprachen zu untersuchen. Othmar Plöckinger hat dafür in dem vorliegenden Band Autoren für 11 Sprachen gefunden, für acht europäische sowie für das Türkische, das Japanische und das moderne Hebräisch. Da er keine besonderen Vorgaben gemacht hat, liegt der Akzent nicht in allen Essays auf denselben Fragestellungen. Neben rein übersetzungstechnischen, sich an der Transition der Sprache orientierenden Beiträgen stehen andere mit einem eindeutig historisch-politischen Akzent. Verlagsgeschichtliche Untersuchungen stehen editionsgeschichtlichen gegenüber. Nur in dem Beitrag über Frankreich wird einmal der Historiker Johann Chapoutot zitiert, der die problematische Hitlerzentrierung der Konzentration auf „Mein Kampf“" kritisiert, über welche die NS-Forschung eigentlich längst hinweg ist.

W. Schieder $(\bowtie)$

Universität zu Köln, Köln, Deutschland

E-Mail: wolfg.schieder@gmx.de 